\title{
ESTUDO TEÓRICO-EXPERIMENTAL DA REMOÇÃO DE DIAZEPAM EMPREGANDO ÓXIDO DE GRAFENO ${ }^{1}$
}

\author{
THEORETICAL-EXPERIMENTAL STUDY APLYING \\ GRAPHENE OXIDE IN THE REMOTION OF DIAZEPAM
}

\section{Taís Alice Junges ${ }^{2}$, Ingrid Rosales Costa ${ }^{3}$, Cristiano Rodrigo Bohn Rhoden ${ }^{4}$, Jussane Rossato ${ }^{5}$ e Sergio Roberto Mortari ${ }^{6}$}

\section{RESUMO}

Efluentes emergentes contribuem cada vez mais na contaminação dos recursos hídricos, um bem esgotável, acarretando problemas ambientais e na saúde pública. Este estudo visa avaliar a remoção do fármaco Diazepam empregando óxido de grafeno (GO) em protocolos de adsorção, por meio de cálculos teóricos e dados experimentais. O GO utilizado nesse trabalho foi obtido pelo método de Hummers e caracterizado pelas técnicas de Difração de Raios-X, Espectroscopia no Infravermelho por Transformada de Fourier e Microscopia Eletrônica de Varredura - MEV. Os resultados, tanto teórico quanto experimentais, mostraram uma interação química entre Diazepam e óxido de grafeno, confirmando que GO pode ser utilizado como adsorvente para Diazepam.

Palavras-chave: Benzodiazepinas, Nanomaterial de Carbono, Simulação Computacional, Cinética.

\section{ABSTRACT}

Emerging effluents increasingly contribute to the contamination of water resources, an exhaustible resource, causing environmental and public health problems. This study aims to evaluate the remotion of the pharmaceutical Diazepam applying graphene oxide (GO) in adsorption protocols, through theoretical calculations and experimental data. The GO used in this work was obtained by the Hummers method and characterized by the techniques of X-ray Diffraction, Fourier Transformed Infrared Spectroscopy and Scanning Electron Microscopy - SEM. The results, both theoretical and experimental, showed a chemical interaction between Diazepam and graphene oxide, confirming that GO can be used as an adsorbent for Diazepam.

Keywords: Benzodiazepines, Carbon nanomaterial, Computational simulation, Kinetic.

\footnotetext{
${ }^{1}$ Trabalho Final de Graduação em Engenharia Química - UFN.

${ }^{2}$ Curso de Engenharia Química - Universidade Franciscana - UFN / BRF, Unidade Marau/RS. E-mail: taisajunges@gmail.com

${ }^{3}$ Acadêmica do Curso de Engenharia Biomédica - Universidade Franciscana. E-mail: ingrid.v.12@gmail.com

${ }^{4}$ Colaborador, Professor do Curso de Engenharia Química - Universidade Franciscana - UFN. E-mail: cristianorbr@ufn.edu.br

${ }^{5}$ Co-orientadora, Professora do Curso de Física Médica - UFN. E-mail: jussaner@gmail.com

${ }^{6}$ Orientador, Professor do Curso de Engenharia Química - UFN. E-mail: mortari@ufn.edu.br
} 


\section{INTRODUÇÃO}

Os primeiros relatos de contaminação de fármacos nos recursos hídricos foram na década de 60, porém apenas em meados de 1990 começaram os estudos neste assunto, em virtude de possíveis alterações no ecossistema de animais aquáticos e pela toxicidade destes compostos (CALISTO; ESTEVES, 2009). Sabe-se que os métodos convencionais de tratamento de esgoto, atualmente empregados nas estações de tratamento de esgotos (ETEs), são ineficientes na degradação e retenção de uma grande variedade de substâncias químicas, chegam aos recursos hídricos na forma integra como em metabólitos e acabam sendo consumidas, tanto por humanos como por animais.

Segundo a ANVISA e disponibilizado pelo site R7: "os brasileiros compraram, em 2018, mais de 56,6 milhões de caixas de medicamentos para ansiedade e para dormir. Foram cerca de 6.471 caixas vendidas por hora ou, aproximadamente, 1,4 bilhão de comprimidos em um ano”. Dentre estes o Diazepam $\left(\mathrm{C}_{16} \mathrm{H}_{13} \mathrm{ClN}_{2} \mathrm{O}\right)$, pertencente ao grupo dos benzodiazepínicos. Este fármaco atua sobre o sistema nervoso central, tendo como principal função controlar os sintomas da ansiedade, convulsões e insônia (KOROLKOVAS, 2015). Conforme Yalkowsky e Yan (2003) a sua solubilidade em água é baixa, cerca de $66 \mathrm{mg} \mathrm{L}^{-1}$ a $25^{\circ} \mathrm{C}$. Porém, na presença de luz solar se tornam bioacumuláveis e tóxicos para o meio hídrico (WEST; ROWLAND, 2012). Cabeza e colaboradores (2012), avaliaram a presença de Diazepam em 20 estações de tratamento de água residuária e detectaram Diazepam em 8 delas. Böger e colaboradores (2018), quantificaram a presença de Diazepam em água de rio urbano, encontrando concentrações de $0,856 \mu \mathrm{g} \mathrm{L^{-1 }}$, utilizando a técnica de SPE-HPLC-DAD.

Com o crescente consumo de medicamentos, o interesse dos grupos de pesquisa no desenvolvimento de novos procedimentos, visando a remoção de fármacos e seus metabólitos no meio ambiente, é de extrema importância. Uma metodologia é o uso da adsorção (JAURIS et al., 2015; PROVENZE et al., 2020; DE SOUZA COUTO et al., 2020), onde os átomos, moléculas ou íons são retidos na superfície de um sólido, através de interações de natureza física ou química. O fármaco (líquido), denominado adsorvato, irá aderir ao material sólido, denominado de adsorvente (FOUST, 1982). O método de adsorção é eficaz, de fácil operação e baixo custo, podendo ser utilizado em processos contínuos e descontínuos (DABROWSKI, 2001; MACHADO et al., 2012).

Não obstante, o método de adsorção pode ser realizado através de diferentes materiais à base de carbono, como o carvão ativado, derivados de grafeno e com nanotubos de carbono (CHOWDHURY et al., 2014). Estes apresentam propriedades como estabilidade química, diversidade estrutural, baixa densidade, estrutura altamente porosa e grande área superficial (GUPTA; SUHAS, 2009; MALAVIYA; SINGH, 2011). Porém a utilização do carvão ativado vem sendo bastante criticada por apresentar baixa estabilidade mecânica e por não serem aplicados em escala industrial (CRINI, 2006).

De acordo com Chowdhury e Balasubramanian (2014) os materiais derivados do grafeno, como óxido de grafeno (GO) e óxido de grafeno reduzido (rGO) são excelentes adsorventes de 
fármacos em recursos hídricos, devido as suas propriedades. A presença de grupos funcionais, como o epóxi, hidroxila, carbonila e carboxila, todos contendo oxigênio em sua estrutura, auxiliam nas interações entre o adsorvente e o adsorvato, como é o caso do óxido de grafeno (JAURIS et al., 2016).

A literatura disponibiliza poucos estudos teórico-experimental, utilizando óxido de grafeno para a remoção de fármacos, em especial o Diazepam em recursos hídricos. Estudos teóricos garantem uma melhor credibilidade ao estudo experimental. Neste sentido, o objetivo deste estudo foi avaliar a adsorção de Diazepam em óxido de grafeno (GO) através de estudo teórico-experimental.

\section{MATERIAIS E MÉTODOS}

\section{REAGENTES QUIIMICOS}

Para a obtenção do óxido de grafeno utilizou-se os seguintes reagentes: Nitrato de Sódio (Nuclear, p. a.), Permanganato de Potássio (Vetec, p. a.), Ácido Sulfúrico (Synth, p. a.), Ácido Nítrico (Fmaia), Peróxido de hidrogênio (30\%) (Neon, p. a.), Ácido Clorídrico (Synth, p. a.), Álcool Etílico (Synth, p. a.) e água ultrapurificada (Milliq®). A substância de trabalho Diazepam, foi adquirida em farmácia de manipulação, no comércio local de Santa Maria-RS.

\section{SÍNTESE DOS MATERIAIS A BASE DE CARBONO E PREPARO DA SOLUÇÃO}

O óxido de grafeno foi obtido através do método de Hummers e Offeman (1958), com adaptação de Moraes e colaboradores (2015) e Rhoden et al. (2017). No procedimento (etapa 1) utilizou-se uma mistura de grafite em flocos (Sigma Aldrich) e $\mathrm{NaNO}_{3}$ contendo 5,0 g, respectivamente. Para a oxidação, a esta mistura adicionou-se $200,0 \mathrm{~mL}$ de $\mathrm{HNO}_{3} / \mathrm{H}_{2} \mathrm{SO}_{4}$, na proporção $1: 3$, ambos concentrados (14 e $18 \mathrm{~mol} \mathrm{~L}^{-1}$, respectivamente). Este processo se deu lentamente e em banho de gelo, sob agitação magnética $(80 \mathrm{rpm})$. Posteriormente, foi adicionado, lentamente, 25,0 g de $\mathrm{KMnO}_{4}$ sob agitação constante, durante 2 horas, sempre em banho de gelo. Após o produto obtido foi aquecido a $60{ }^{\circ} \mathrm{C}$ durante 30 minutos, sob agitação magnética. Passado o tempo de aquecimento, adicionou-se $37,0 \mathrm{~mL}$ de $\mathrm{H}_{2} \mathrm{O}_{2}$ (30\%). Na sequência, foram adicionados 50,0 mL de solução de $\mathrm{HCl} 10 \%$ (v/v) e 45,0 mL de água destilada congelada (gelo). Essa mistura permaneceu em geladeira $\left(4^{\circ} \mathrm{C}\right)$ durante 24 horas (etapa 2). O sobrenadante obtido foi descartado, e o sólido lavado várias vezes com água, obtendo-se assim o óxido de grafite. O óxido de grafite obtido foi sonicado por duas horas obtendo-se assim o óxido de grafeno (etapa 3). O sobrenadante castanho claro foi recolhido, e o óxido de grafeno separado por filtração. Após o material foi seco em estufa $\left(80^{\circ} \mathrm{C}\right)$ por 24 horas e armazenado em frascos de polipropileno (etapa 4). A figura 1 apresenta as etapas envolvidas no processo. 
Figura 1 - Representação esquemática da obtenção do óxido de grafeno

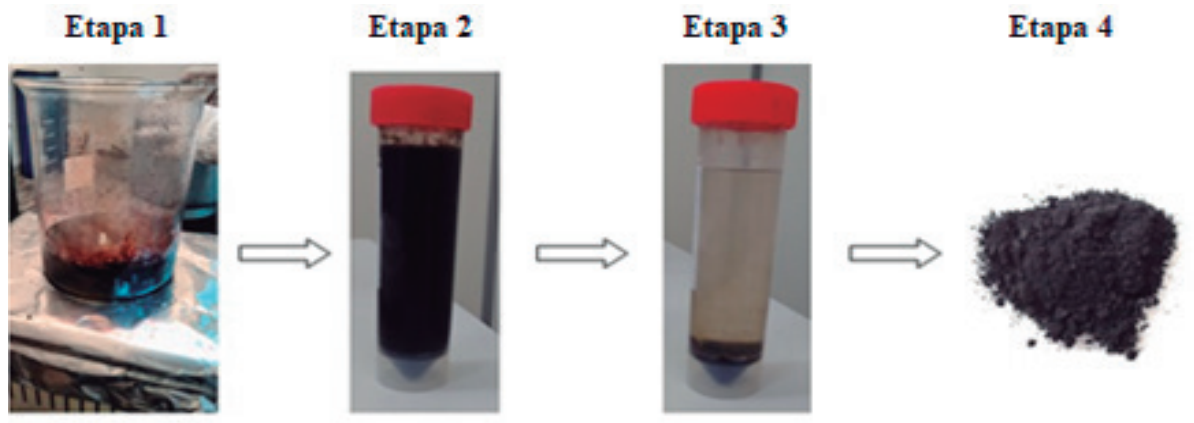

Fonte: Construção do autor

\section{CARACTERIZAÇÃO}

O óxido de grafeno obtido foi caracterizado pelas técnicas de Difração de Raios- X (D2 Phaser, Bruker), Espectroscopia no Infravermelho (FTIR, Spectro One, Perkin Elmer), e Microscopia Eletrônica de Varredura - MEV (MEV/FEG - SIGMA VP, Carl Zeiss Microscopy).

\section{ESTUDO DE ADSORÇÃO}

O estudo foi realizado em batelada para avaliar a capacidade de adsorção do Diazepam pelo óxido de grafeno, conforme a metodologia adaptada (XU; WANG; ZHU, 2012). A solução padrão do adsorvato foi preparada em etanol, com concentração de $28 \mathrm{mg} \mathrm{L}^{-1}$. Esta concentração foi utilizada em função de ter uma boa razão sinal/ruído, em absorbância (espectrofotômetro UV-Vis, 1450 PC, Shimadzu), no comprimento de onda de $230 \mathrm{~nm}$. O espectro eletromagnético é apresentado na figura 2.

Figura 2 - Espectro eletromagnético (200 a $400 \mathrm{~nm}$ ) do Diazepam em meio alcoólico, com pH neutro $(7,0)$ e básico $(8,0)$

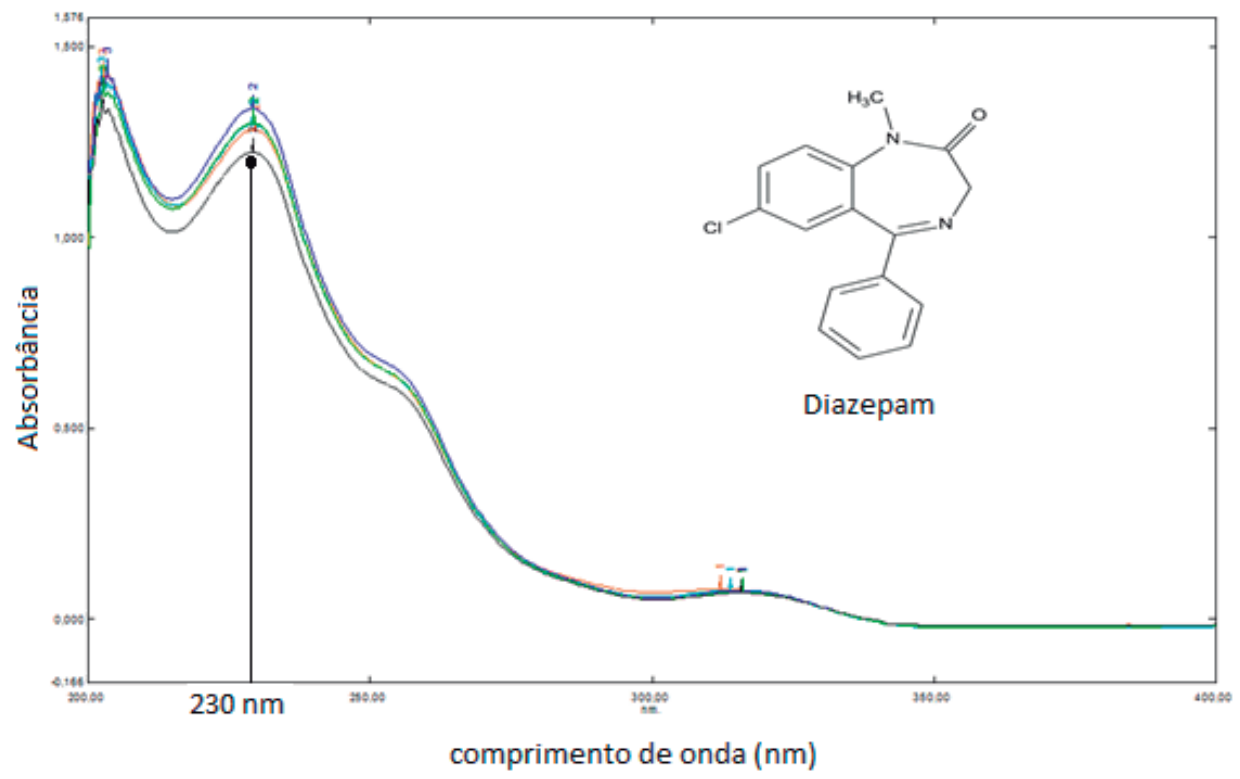

Fonte: Construção do autor 
Em um erlenmayer de $100 \mathrm{~mL}$ foram colocados $250 \mathrm{mg}$ do adsorvente (GO) e 50,0 mL do adsorvato (Diazepam, $28 \mathrm{mg} \mathrm{L}^{-1}$ ). O erlenmayer foi posicionado em uma mesa agitadora, em velocidade de agitação de $200 \mathrm{rpm}$. Alíquotas de 5,0 mL foram retiradas, a cada 10 minutos (até 120 minutos), centrifugadas a $3500 \mathrm{rpm}$ por 5 minutos e levadas ao espectrofotômetro, medidas no comprimento de onda de $230 \mathrm{~nm}$. O pH foi mantido constante $(8,0)$ e a temperatura mantida em $25^{\circ} \mathrm{C}$.

A quantidade do fármaco adsorvido, e a porcentagem de remoção do Diazepam pelo nanoadsorvente, foi calculado mediante aplicação das equações 1 e 2, respectivamente:

$$
\begin{gathered}
q=\frac{\left(C_{0}-C_{f}\right)}{m} x V \\
\% \text { Remoção }=100 \times \frac{\left(C_{0}-C_{f}\right)}{C_{0}}
\end{gathered}
$$

onde, $q$ representa a quantidade de Diazepam adsorvido pelo adsorvente ( $\left.\mathrm{mg} \mathrm{g}^{-1}\right)$; $C o$ a concentração inicial da solução de composto farmacêutico em contato com o adsorvente (mg L-1); $C f$ a concentração do Diazepam após o processo de adsorção $\left(\mathrm{mg} \mathrm{L}^{-1}\right)$; $V$ o volume de solução de fármaco (L) em contato com o adsorvente e $m$ a massa do adsorvente $(\mathrm{g})$.

\section{CÁLCULOS TEÓRICOS}

O estudo teórico baseou-se em cálculos de primeiros princípios, ab initio, que descreve a natureza quântica dos elétrons. Através dos cálculos ab initio foi possível analisar as propriedades eletrônicas e estruturais da interação entre o fármaco ansiolítico, Diazepam, e o nanomaterial, GO. A metodologia existente por trás dos cálculos utiliza uma descrição atomística de natureza quântica, conhecida como Teoria do Funcional da Densidade (Density Functional Theory - DFT), onde as propriedades física ou química de um sistema foram determinadas, a partir sua densidade eletrônica. Essa teoria está associada ao método de pseudopotenciais, em que descreve apenas os elétrons que participam efetivamente das ligações químicas, a qual está implementada no código computacional SIESTA (Spanish Initiative for Electronic Simulations with Thousand of Atoms). Para descrever a interação de troca e correlação, foi utilizado a aproximação da densidade local (Local Density Approximation LDA) (CEPERLEY; ALDER, 1980). A interação entre os elétrons de valência e os elétrons de caroço foi descrita pelos pseudopotenciais de norma conservada de Troullier-Martins (TROULLIER; MARTINS, 1991). Os cálculos teóricos foram realizados no CENAPAD (Centro Nacional de Processamento de Alto Desempenho) disponibilizado pela UNICAMP.

Para calcular a energia de ligação entre o Diazepam e óxido de grafeno, foi utilizada a seguinte equação: 


$$
\mathrm{E}_{\mathrm{L}}=\mathrm{E}_{\mathrm{T}(\text { nanomaterial+Diazepam) }}-\mathrm{E}_{\mathrm{T}(\text { Diazepam })}-\mathrm{E}_{\mathrm{T}(\text { nanomaterial })}(3)
$$

em que o termo é a energia total do sistema, isto é, a energia total do nanomaterial interagindo com o Diazepam. Já o termo representa a energia total do Diazepam e é a energia total do nanomaterial.

O óxido de grafeno (GO) possui abundância de grupos funcionais hidrofílicos, como hidroxila e epóxi no plano basal, e grupos carboxílicos e carbonilas na periferia do plano. Já o óxido de grafeno reduzido (rGO) possui poucos grupos funcionais, mais precisamente, apenas hidroxilas (HE et al. 2017). Por este fator, a capacidade adsortiva é menor no rGO, pois não há muitos grupos funcionais para a adsorção. Essa definição entre os nanomateriais é válida tanto teoricamente como experimentalmente. $\mathrm{O}$ adsorvente criado computacionalmente foi composto por: 144 átomos de carbono (C), mais quatro diferentes grupos funcionais, um par de carbonilas (C-CHO), um par de carboxilas (C-COOH), um par de hidroxilas $(\mathrm{C}-\mathrm{OH})$ e um par de epóxi (C-O-C).

\section{RESULTADOS}

\section{CÁLCULOS TEÓRICOS}

De acordo com Junges e colaboradores (2015), a propriedade eletrônica do fármaco (diferença entre Homo e Lumo) foi de 2,91 eV e seus plots de densidade eletrônica de carga, estão localizados sobre os átomos de cloro, nitrogênio, oxigênio e sobre os anéis aromáticos, contendo o nitrogênio e cloro, conforme mostra a figura 3. No óxido de grafeno, as propriedades eletrônicas, diferença entre o gap varia de $0,02 \mathrm{eV}$ e suas concentrações de carga encontram-se sobre os grupos funcionais de acordo com a figura 4 .

Figura 3- Estrutura otimizada, níveis de energia e densidade de carga do Diazepam

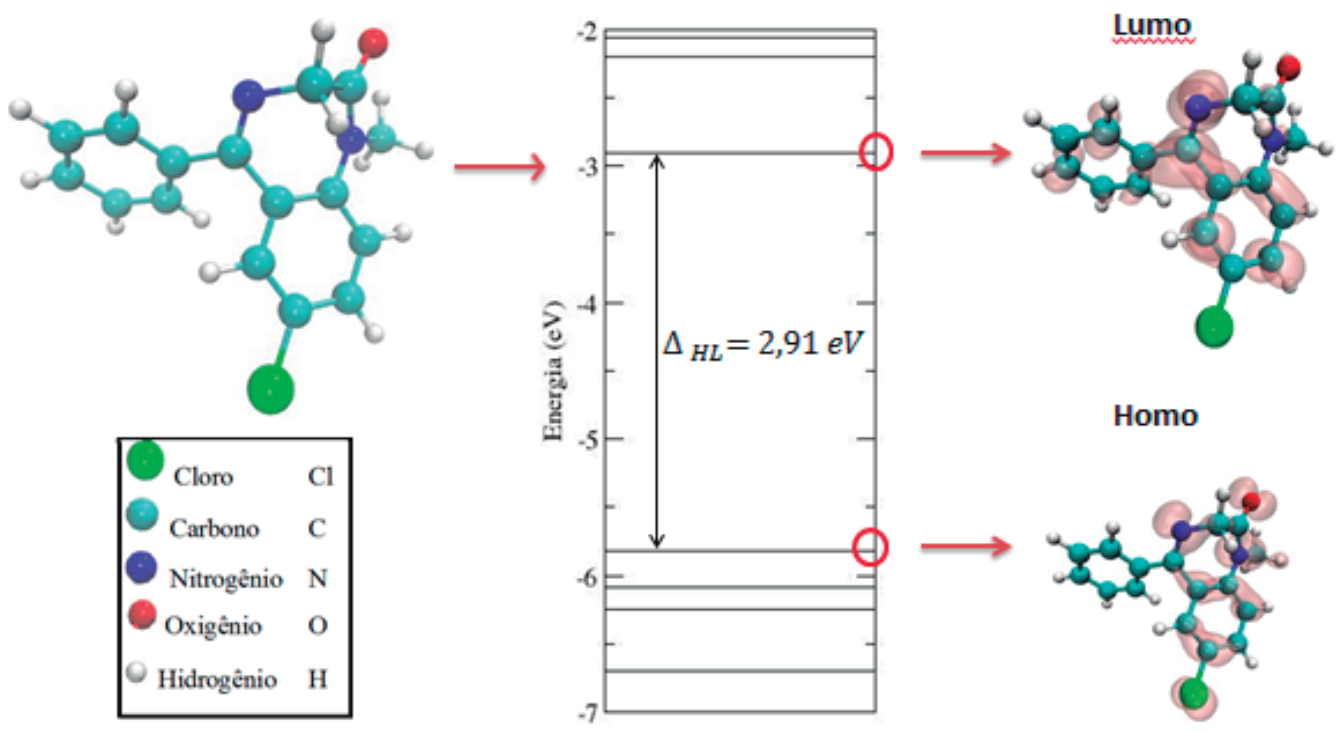

Fonte: (JUNGES; JAURIUS; ROSSATO, 2015) 
As informações citadas estão demonstradas nas figuras 4 e 5. Em 4(a) a estrutura do óxido de grafeno, em 4(b) as estruturas de banda e em 4(c) os plots de densidade eletrônica de carga.

Figura 4 - Estrutura otimizada do óxido de grafeno em (a), estruturas de bandas em (b) e densidade eletrônica de carga em (c)

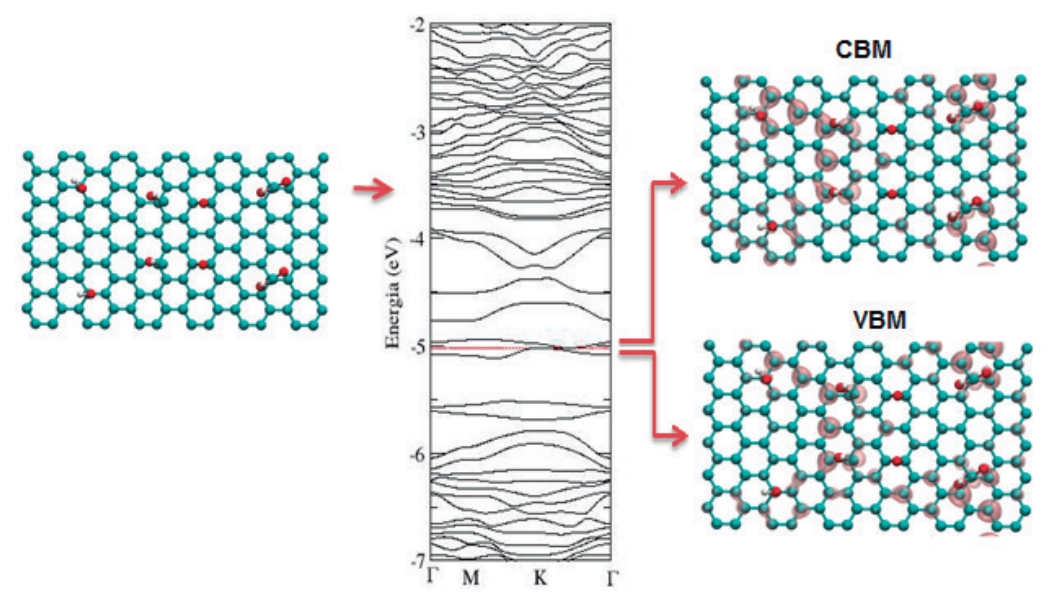

Fonte: Construção do autor

As interações entre o fármaco e o GO foram realizadas através dos átomos de cloro, nitrogênio, oxigênio e átomos de carbono do anel aromático com todos os grupos funcionais do GO, totalizando em 12 interações. A interação mais estável ocorreu através do átomo de nitrogênio do Diazepam com o grupo carbonila do GO, resultando em uma energia de ligação de $-3,37 \mathrm{eV}$. A partir da figura 5, observou-se em (a) a quebra da ligação entre os átomos C-C e O-H do grupo carbonila do GO e a formação de uma ligação química entre o átomo de hidrogênio da carbonila com o átomo de nitrogênio do Diazepam. Em (b) representação dos níveis de energia do Diazepam, e as estruturas de bandas do GO e da interação mais estável (COH-N), respectivamente, ressaltando que as propriedades eletrônicas têm uma diferença entre VBM e CBM de 0,04 eV e em (c) os plots de densidade eletrônica de carga para o topo da banda de valência (VBM) e o fundo da banda de condução (CBM) para essa interação.

Figura 5- Interação entre o átomo de nitrogênio do Diazepam com o grupo funcional carbonila do GO. Em (a) estrutura da interação otimizada, (b) níveis e estruturas de banda e em (c) plots de densidade eletrônica de carga

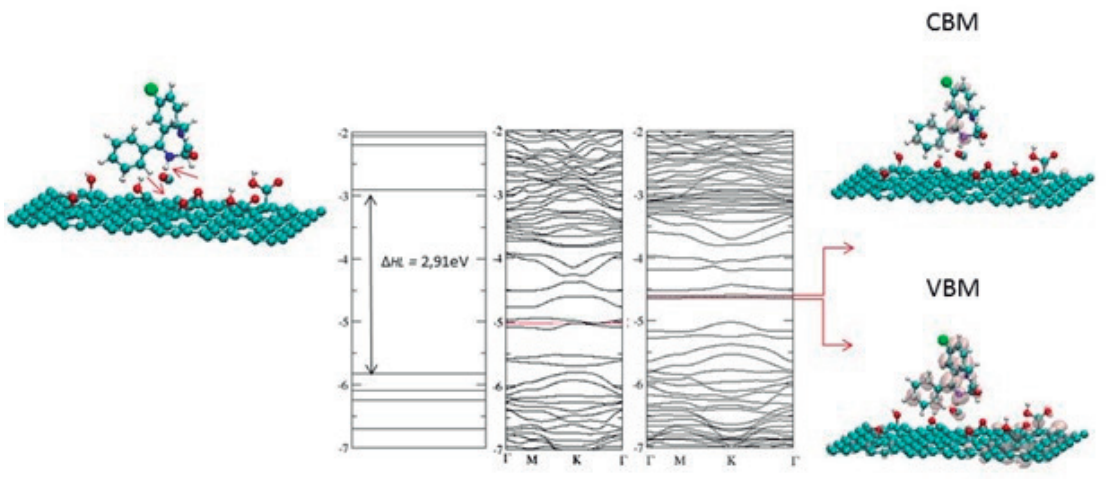


Com a realização dos plots de densidade eletrônica de carga para a interação mais estável observou-se que para VBM as cargas estão concentradas sobre o GO e sobre o Diazepam e para o CBM as cargas estão em maior concentração sobre o fármaco. Por meio destes resultados, conclui-se pela possibilidade de ocorrência de adsorção química no sistema GO/Diazepam. Para confirmar os cálculos aplicou-se o sistema teorizado em um protocolo de adsorção.

\section{CARACTERIZAÇÃO DO ADSORVENTE}

No difratograma de Raios-X, apresentado na figura 6(a), pode-se observar a presença dos pi$\cos 2 \Theta \approx 11,10^{\circ}$ e em $42,32^{\circ}$, indicando a oxidação do material grafítico, podendo-se ainda observar em $2 \Theta \approx 25^{\circ}$ um residual do grafite empregado (LUJANIENÉ et al., 2016).

Na figura 6(b), está representado o FTIR do GO produzido neste trabalho. Observa-se uma banda expressiva em torno de $3480 \mathrm{~cm}^{-1}$, referindo-se a deformação axial do grupo hidroxila, mostrando a presença do grupo - $\mathrm{OH}$ nas superfícies do nanomaterial. A frequência de estiramento da ligação dupla carbono-oxigênio $\left(\mathrm{R}_{2} \mathrm{C}=\mathrm{O}\right)$ apresenta um pico em $1617 \mathrm{~cm}^{-1}$ indicando a presença dos ácidos carboxílicos. As bandas remanescentes, que aparecem entre $1497-1452 \mathrm{~cm}^{-1}$, relacionam-se ao estiramento ou deformação axial das ligações C=C (RAMANATHAN et al., 2005).

Figura 6 - Óxido Grafeno produzido neste trabalho: (a) Difratograma de raios-X; (b) Espectro de FTIR
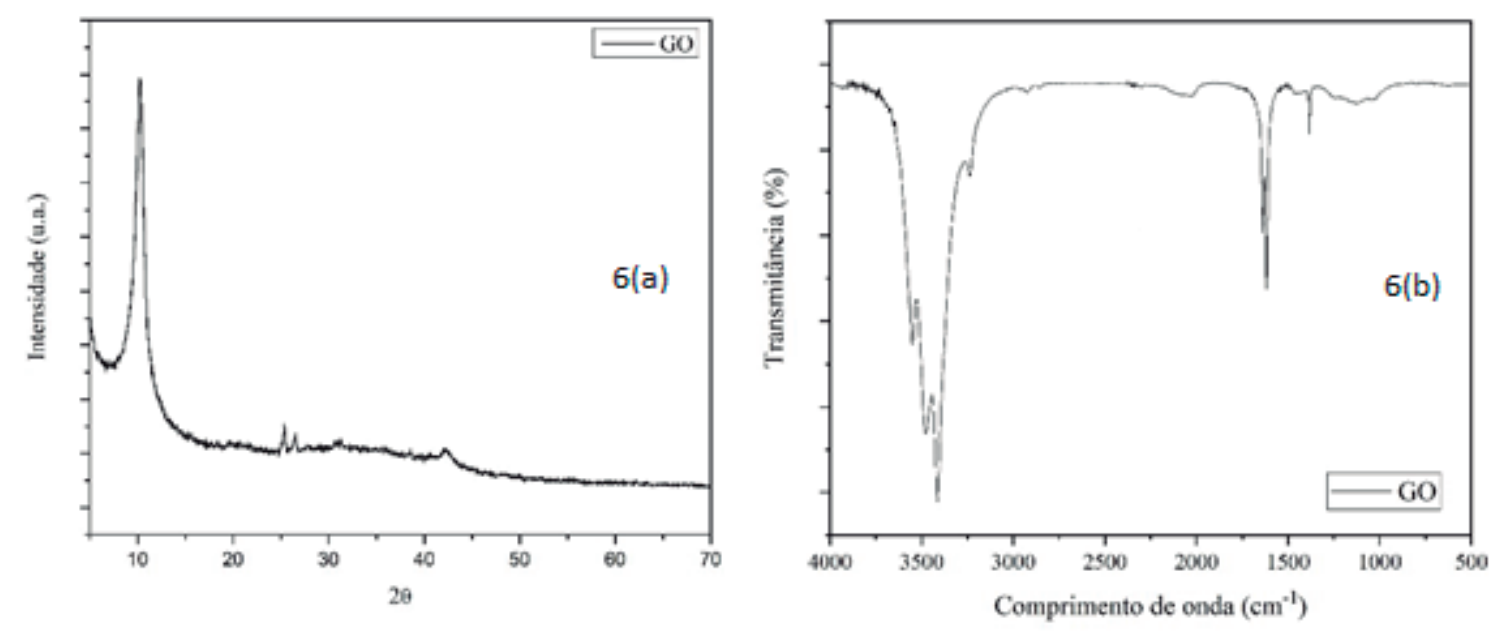

Fonte: Construção do autor

A morfologia do óxido de grafeno produzido, é apresentada na figura 7 por imagem de microscopia eletrônica de varredura. Percebe-se as folhas obtidas pela esfoliação química do grafite (RHODEN, 2018). 
Figura 7 - Imagem de Microscopia Eletrônica de Varredura (MEV-FEG),

do óxido de grafeno produzido neste trabalho. Sem recobrimento da amostra

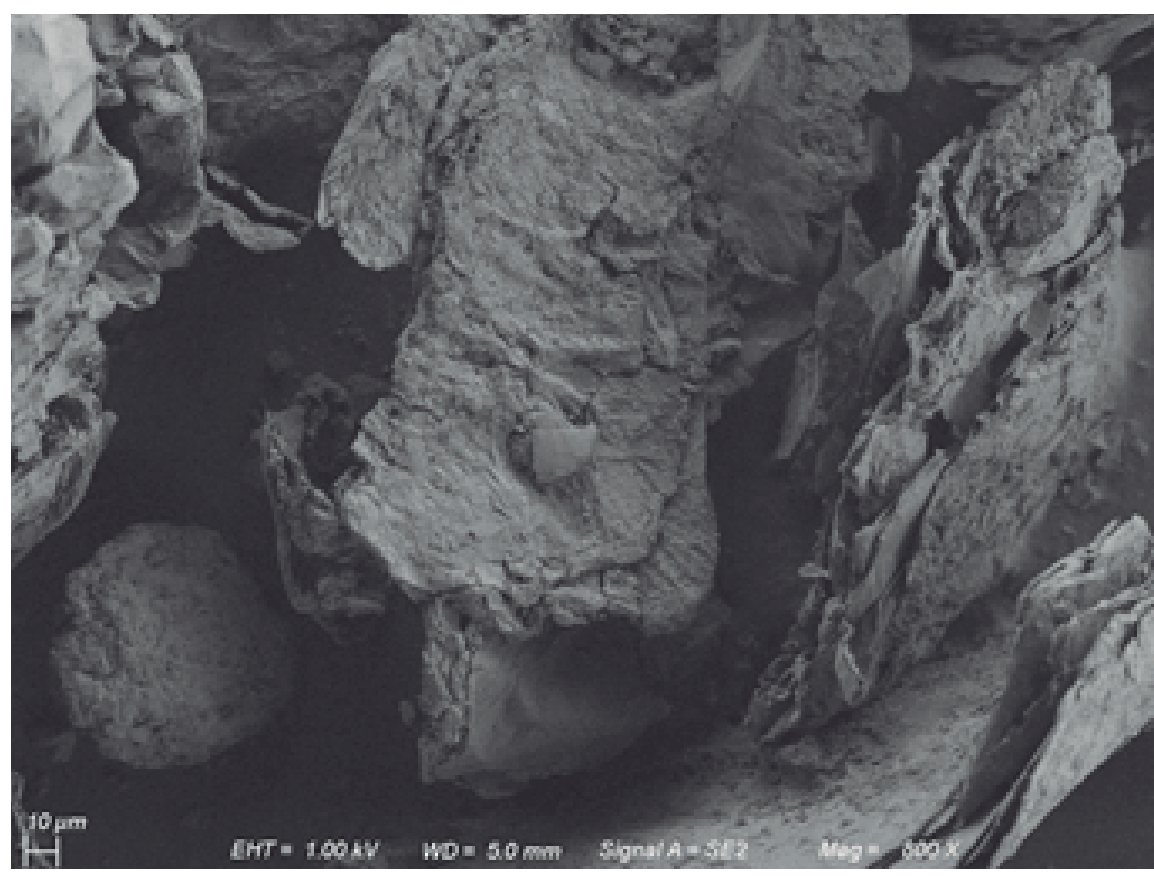

Fonte: Construção do autor

\section{ESTUDOS DE ADSORÇÃO}

Para avaliar a adsorção do Diazepam em GO, tendo como variáveis a massa do adsorvato, massa do adsorvente e o pH do meio (sistema avaliado em meio ácido, neutro e básico). Os resultados indicaram que uma massa de $50 \mathrm{mg}$ de adsorvente (GO) foi insuficiente, ou seja, o adsorvente saturava em menos de 5 minutos. Para uma massa de $150 \mathrm{mg}$ de adsorvente (GO) observou-se que após agitação por 10 minutos ocorreu a saturação do adsorvente. Já em uma massa de adsorvente de 250 mg os resultados foram satisfatórios. Com isso neste trabalho adotou-se, para uma solução de 10,0 mL de Diazepam com concentração de $28 \mathrm{mg} \mathrm{L}^{-1}, 250 \mathrm{mg}$ de adsorvente (GO).

Após o tempo de agitação (até 80 minutos, com avaliações de 10 em 10 minutos) alíquotas de $5,0 \mathrm{~mL}$ eram retiradas e centrifugadas por 5 minutos a $3500 \mathrm{rpm}$ e então realizadas as medidas de absorbância.

A curva da cinética de adsorção do Diazepam em óxido de grafeno está representada na figura 8 , onde pode-se verificar que a concentração inicial vai diminuindo em função do tempo, indicando que a cinética de adsorção pode ser modelada matematicamente pelos modelos cinéticos. 
Figura 8 - Cinética de adsorção do Diazepam em óxido de grafeno

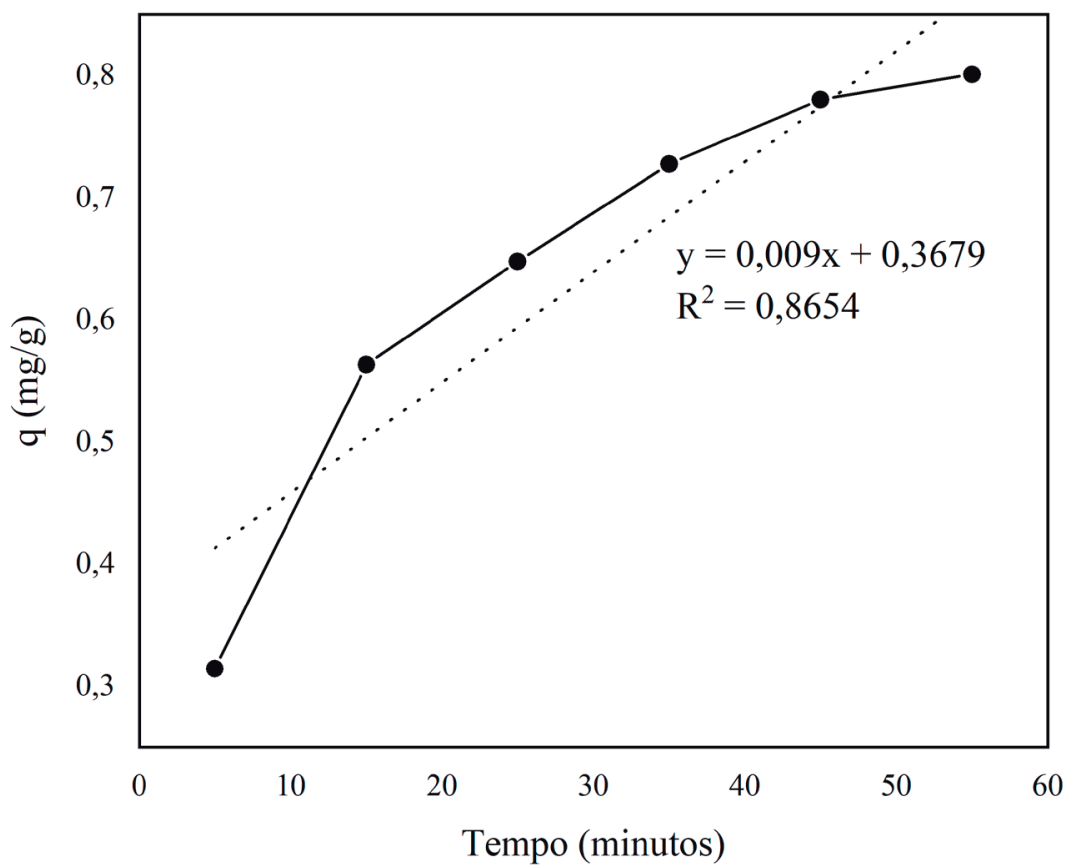

O cálculo para avaliar a capacidade adsortiva após 80 minutos, foi:

Capacidade de adsorção $=(($ Cef x 100) x Ceo $)-100 \%$

onde Cef representa a concentração final do processo de adsorção e Ceo a concentração inicial do processo de adsorção. Logo, ao final dos 80 minutos foi adsorvido apenas 14\% do Diazepam em óxido de grafeno. De acordo com observado pôde-se auferir uma remoção de $86 \%$ do fármaco contaminante da amostra. Estes resultados corroboram o modelo teórico desenvolvido, oferecendo o óxido de grafeno como excelente opção de adsorvente em sistemas aquosos.

\section{CONCLUSÃO}

Através dos resultados obtidos neste estudo foi possível observar que a adsorção do Diazepam pelo GO ocorreu de maneira efetiva podendo ser modelada matematicamente pelos modelos cinéticos. De acordo com a simulação teórica o sistema mais estável possui uma energia de ligação de $-3,37 \mathrm{eV}$. Também, observou-se a quebra da ligação entre os átomos C-C e O-H do grupo carbonila do GO e a formação de uma ligação química entre o átomo de hidrogênio da carbonila com o átomo de nitrogênio do Diazepam, característica fundamental de uma interação química. Os resultados experimentais corroboraram com os cálculos teóricos confirmando a eficiência do protocolo de adsorção aplicando com a remocão de $86 \%$ do contaminante. Considerando o modelo de adsorção química observado, novos estudos estão sendo conduzidos objetivando a dessorção e consequente reaproveitamento do adsorbato. 
AGRADECIMENTOS

PROPESQ/UFN; FAPERGS

\section{REFERÊNCIAS}

BÖGER, B. et al. Determination of carbamazepine and Diazepam by SPE-HPLC-DAD in Belém River water, Curitiba-PR/Brazil. Revista Ambiente \& Água, v. 13, n. 2, 2018.

CABEZA, Y. et al. Monitoring the occurrence of emerging contaminants in treated wastewater and groundwater between 2008 and 2010. The Baix Llobregat (Barcelona, Spain). Journal of Hazardous Materials, v. 239, p. 32-39, 2012.

CALISTO, V.; ESTEVES, V. I. Psychiatric pharmaceuticals in the environment. Chemosphere, v. 77, n. 10, p. 1257-1274, 2009.

CEPERLEY, D. M.; ALDER, B. J. Ground State of the Electron Gas by a Stochastic Method. Physical Review Letters, v. 45, p. 566-569, 1980.

CHOWDHURY, S.; BALASUBRAMANIAN, R. Recent advances in the use of graphene-family nanoadsorbents for removal of toxic pollutants from wastewater. Advances in Colloid and Interface Science, v. 204, p. 35-56, 2014.

CRINI, G. Non-conventional low-cost adsorbents for dye removal: a review. Bioresource technology, v. 97, p. 1061-1085, 2006.

DABROWSKI, A.; Adsorption - from theory to practice. Advances in colloid and interface science, v. 93, p. 135-224, 2001.

DE SOUZA COUTO, J. M. et al. Remoção de Carbamazepina em soluções aquosas por adsorção em carvão ativado em pó e avaliação da toxicidade crônica/Carbamazepine removal in aqueous solutions by adsorption into powder activated carbon and chronic toxicity assessment. Brazilian Journal of Development, v. 6, n. 2, p. 8744-8765, 2020.

FOUST. S. A. et al., Princípios das Operações Unitárias, 2. Ed. LTC - Livros Técnicos e Científicos, RJ, 1982. 
GUPTA, V. K. et al. Low-cost adsorbents: Growing approach to wastewater treatment - a 20 review. Critical reviews in environmental science and technology, v. 39, p. 783-842, 2009.

HE, K. et al. Stability, transport and ecosystem effects of graphene in water and soil environments. Nanoscale, v. 9, n. 17, p. 5370-5388, 2017.

HUMMERS JR, W. S; OFFEMAN, R. E. Preparation of graphitic oxide. Journal of the American Chemical Society, v. 80, n. 6, p. 1339-1339, 1958.

JAURIS, I. M. et al. Adsorção do fármaco nimesulida em nanoestruturas de carbono. Disciplinarum Scientia. v. 16, n. 2, p. 245-256, 2015.

JAURIS, I. M. et al. Adsorption of sodium diclofenac on graphene: a combined experimental and theoretical study. Physical Chemistry Chemical Physics, v. 18, n. 3, p. 1526-1536, 2016.

JUNGES, T. A.; JAURIS, I. M.; ROSSATO, J. Adsorção de Diazepam com óxido de grafeno: Uma abordagem de primeiros princípios. Disciplinarum Scientia| Naturais e Tecnológicas, v. 16, n. 2, p. $151-160,2015$.

KOROLKOVAS, A.; Dicionário Terapêutico Nacional. 21. ed. Rio de Janeiro: Guanabara Koogan, 2015.

LUJANIENĖ, G. et al. Sorption of radionuclides and metals to graphene oxide and magnetic graphene oxide. Journal of Radioanalytical and Nuclear Chemistry, v. 307, n. 3, p. 2267-2275, 2016.

MACHADO, F. M. et.al. Adsorption of Reactive Blue 4 dye from water solutions by carbon nanotubes: Experiment and theory. Physical Chemistry Chemical Physics, v. 14, p. 11139-11153, 2012.

MALAVIYA, P. ; SINGH, A. Physicochemical technologies for remediation of chromiumcontainig waters and wastewaters. Critical reviews in environmental science and technology, v. 41, p. 1111-1172, 2011.

MORAES, F. C. et al. Sensitive determination of $17 \beta$-estradiol in river water using a graphene based electrochemical sensor. Analytica chimica acta, v. 881, p. 37-43, 2015.

PROVENZI, E. L. D. et al. Estudo da Adsorção de Tetraciclina em uma Matriz de Poliuretano Expandido com Carvão Ativado de Casca de Banana. Revista Tecnológica, v. 29, n. 2, p. 430-435, 2020. 
RAMANATHAN, T.; FISHER, F. T.; RUOFF, R. S.; BRINSON, L. C. Amino-Functionalized Carbon Nanotubes for Binding to Polymers and Biological Systems. Chem. Mater, v. 17, p. 1290-1295, 2005.

RHODEN, C. R. B. et al. Síntese Fácil e Direta do Óxido de Grafeno Magnético. Disciplinarum Scientia, v. 18, n. 2, p. 389-397, 2017.

RHODEN, C. R. B. Síntese de análogos de peptídeos incorporados a materiais nanoparticulados de carbono funcionalizados via reações multicomponentes. 2018. 107f. Tese de Doutorado (Doutor em Nanociências), Universidade Franciscana, 2018.

TROULLIER, N.; MARTINS, J. L. Efficient pseudopotentials for plane-wave calculations. Physical Review. B, v. 43, p. 1993-2006, 1991.

XU, J.; WANG, L.; ZHU, Y. Decontamination of bisphenol A from aqueous solution by graphene adsorption. Langmuir, v. 28, p. 8418-8425, 2012.

WEST, C. E.; ROWLAND, S. J. Aqueous Phototransformation of Diazepam and Related Human Metabolites under Simulated Sunlight. Reino Unido: ACS publication, 2012.

YALKOWSKY, S.H.; YAN, H. Handbook of Aqueous Solubility Data: An Extensive Compilation of Aqueous Solubility Data for Organic Compounds. Extracted from the AQUASOL database. Boca Raton: CRC Press LLC, p. 1031, 2003. 
